\title{
14. THE STELLAR VELOCITY FIELD IN M 51
}

\author{
S. M. SIMKIN \\ Columbia University, New York, N.Y., U.S.A., and \\ Kitt Peak National Observatory*, Tucson, Ariz., U.S.A.
}

\begin{abstract}
Radial velocities have been measured from the absorption lines on two image tube spectra of M 51. These velocities show large deviations from the 'smoothed' rotation curve for that object. The measurements seem to indicate that both the stars and the gas moye in the same way.
\end{abstract}

At present, almost all of our information about the velocity fields in external spiral galaxies comes from observations of their gaseous component. It is now possible, with the help of image tubes, to observe the stellar motions in the disk regions of these objects as well. This note describes the initial results of such observations on M 51 .

In March of 1968 two spectra were obtained of the integrated light from the disk stars in M 51. These were taken with the cassegrain spectrograph, at $100 \AA / \mathrm{mm}$ dispersion, and a Carnegie image tube at the $84^{\prime \prime}$ telescope of Kitt Peak Observatory. The spectrograph slit for each of these plates was in an East-West position, perpendicular to the line of nodes for this object as determined by the Burbidges (1964) and Carranza et al. (1969). Figure 1 shows the slit positions. These were chosen to intercept the 'red' spiral arms, found in composite photographs of this object by Zwicky (1955) and Sharpless and Franz (1963). The slits also cross the well defined 'blue' arms. Thus, the composite spectra are formed primarily from the stars that participate in the spiral structure.

Radial velocities at various positions along the slit were determined by measuring redshifts for the absorption lines $\mathrm{H} \delta, \mathrm{H} \zeta, \mathrm{H} \eta$, and $\mathrm{H} \theta$. The emission lines $\mathrm{H} \beta, \mathrm{H} \gamma$, and $\mathrm{H} \delta$ were also measured where present and reduced separately. The measurements were made on a Mann two-coordinate measuring machine and reduced using a twodimensional dispersion curve, obtained from full slit comparison spectra, which gives corrections for changes in dispersion and scale as a function of both wavelength and slit position. The principal errors in these measurements arise from the difficulty of centering on the lines, which are intrinsically broad and slightly distorted by the large grain size of the image-tube phosphor. The probable errors calculated for velocities obtained at the same position from different lines and those calculated for velocities obtained from several independent measurements of the same line are of the same magnitude and amount to about $60 \mathrm{~km} \mathrm{~s}^{-1}$ for absorption lines and $30 \mathrm{~km} \mathrm{~s}^{-1}$ for emission lines.

The results of these measurements are plotted in Figures 2 and 3. The velocities given are heliocentric but no other corrections have been applied. In the same figures are indicated the expected radial velocities at these positions if the only motions present are circular ones following the average rotation curve for $\mathrm{M} 51$ found by the

* Operated by The Association of Universities for Research in Astronomy, Inc., under contract with the National Science Foundation. 


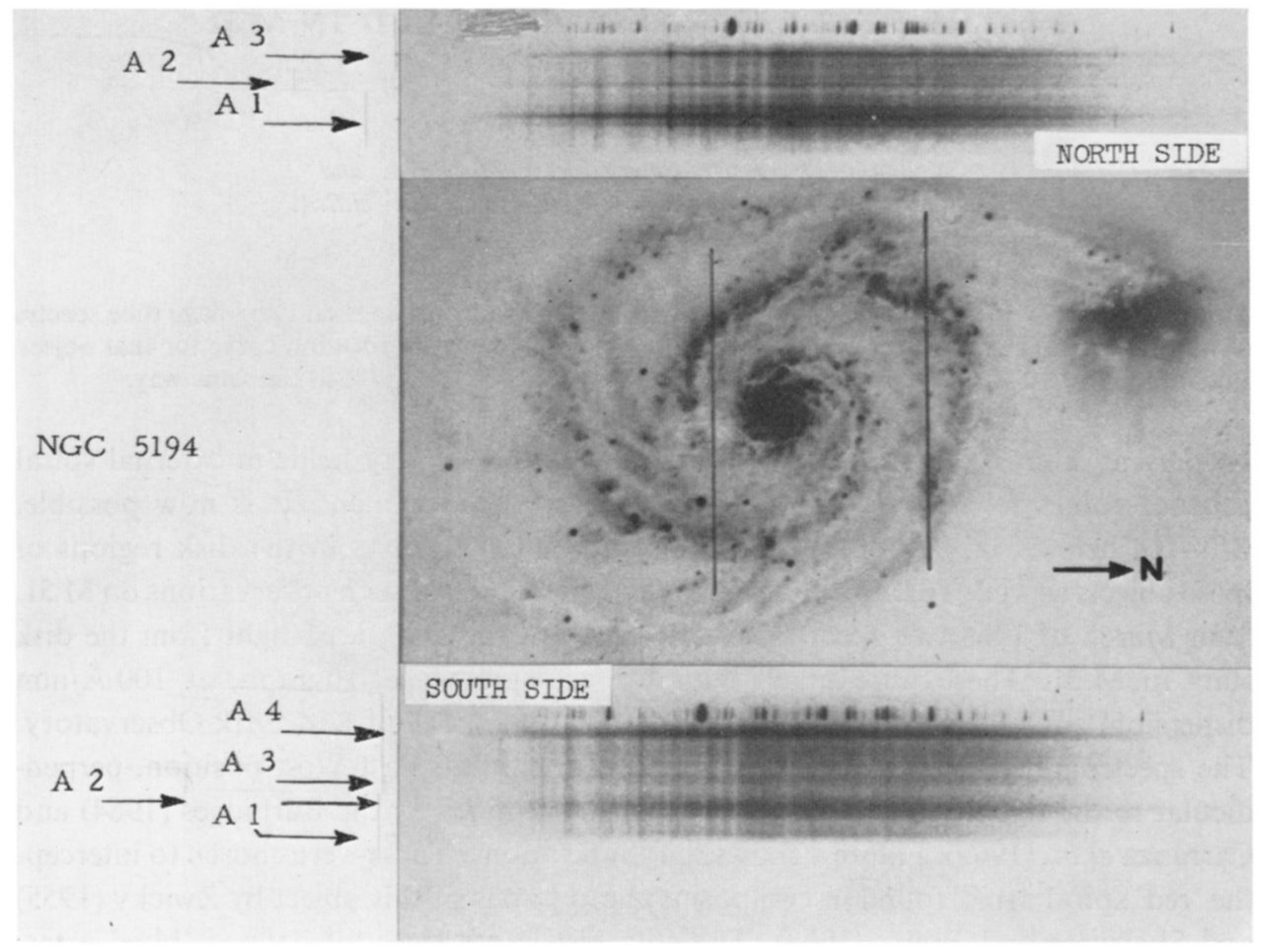

Fig. 1. Spectra and slit positions. The photograph is from a plate taken at Kitt Peak Observatory, April 1968. (103aO + GG 13).

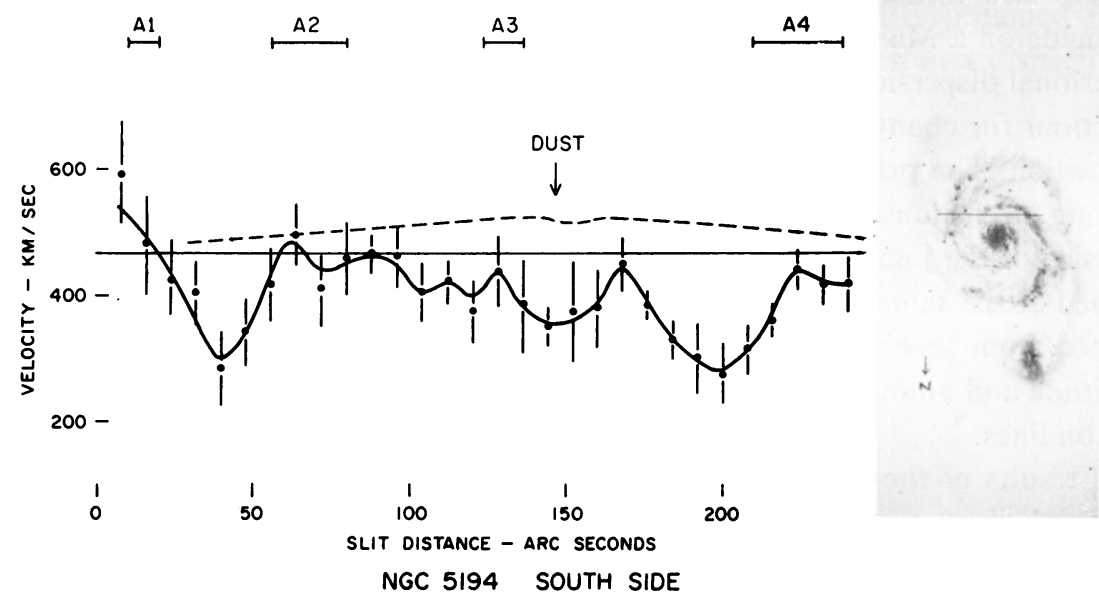

Fig. 2. Absorption line measurements with error bars indicating twice the calculated probable error. The dashed line is the expected velocities, the solid straight line the recessional velocity for the system [Burbidge (1964); Carranza et al. (1969)]. 


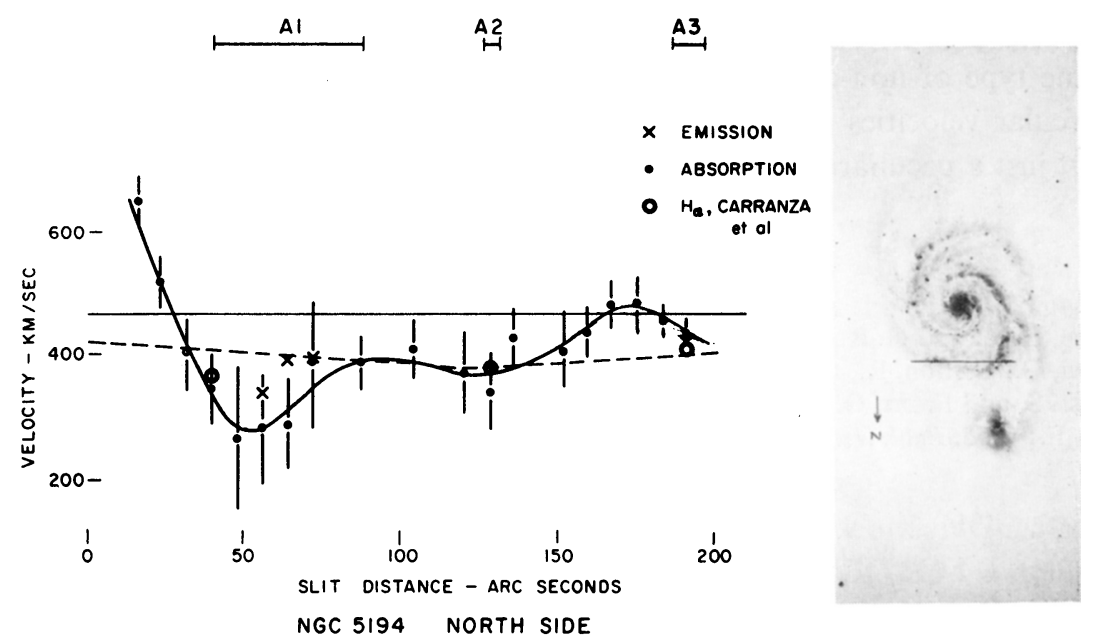

Fig. 3. The symbols as in Figure 2.

Burbidges (1964) and Carranza et al. (1969). In addition, Figure 3 contains some points derived from emission line measurements on the northern spectrum and some points determined by Carranza et al. (1969) using $\mathrm{H} \alpha$ interferometry. In spite of the considerable deviation of the absorption line measurements from the projected 'average circular velocities' at these points, the velocities obtained from the ionized gas evidently agree with those from the stars. The agreement between the present measurements and the observations of Carranza et al. indicates that the absorption line data do not suffer from any large systematic error in the velocity zero point.

Inspection of Figures 2 and 3 suggests two possible conclusions. The most obvious inference is that the motions of the stars as well as the gas in the disk of M 51 show significant deviations from 'average projected circular velocity'. These deviations are not, however, as significant at points on or near the line of nodes. Since the motions of the stars and the gas are similar, they cannot be explained as the result of hydrodynamical effects alone but must be caused by some process that effects both the stellar and the gaseous components of the disk. The lack of notable deviations at the points near the line of nodes may indicate that the non-circular velocities are primarily in the $z$ direction, as found by the Burbidges for the gas near the nucleus. This possibility can only be decided by further observations along the line of the nodes.

The second impression gained from Figures 2 and 3 is that the absorption line velocities tend to be closest to the 'average projected circular velocities' near the outer parts of the blue spiral arms. Clearly this is only a tentative suggestion and also requires further observations.

It is possible that the velocity deviations reported here are the result of the perturbing influence of NGC 5195. There is, however, no direct evidence for this in the present observations. It is, moreover, the author's impression, gained from inspection of lower dispersion spectra of NGC 628 and 1232 and from conversations with 
A. D. Code, that the absorption lines in spectra of other face on, Sc objects exhibit the same type of non-circular velocities as found for M 51. If this is so, then such non-circular velocities may be a general feature of well defined, late spiral systems and not just a peculiarity of M 51 .

\section{References}

Burbidge, E. M. and G. R.: 1964, Astrophys. J. 140, 1445.

Carranza, G., Grillon, R., and Monnet, G.: 1969, Astron. Astrophys. 1, 479.

Sharpless, S. and Franz, O. G.: 1963, Publ. Astron. Soc. Pacific 75, 219.

Zwicky, F.: 1955, Publ. Astron. Soc. Pacific 67, 232. 